\title{
Réduction des pertes de frottement pour des écoulements à surface libre avec entraînement d'air. Augmentation de la débitance d'un coursier d'évacuateur de crues
}

\author{
Hubert Chanson \\ Maître de Conférences en Hydraulique et Mécanique des Fluides \\ Department of Civil Engineering, The University of Queensland, \\ Brisbane QLD 4072, Australie
}

\section{Présentation}

Considérant un écoulement sur un coursier d'évacuateur de crues (fig. l), l'écoulement est initialement peu turbulent. Cependant, une couche limite turbulente se développe au long du radier, jusqu'à ce que la limite supérieure atteigne la surface libre. A partir de là, les vitesses de fluctuations turbulentes sont suffisamment importantes pour entraîner une certaine quantité d'air, dans le cas d'écoulements supercritiques (WISNER 1965), et un écoulement quasi-homogène eau-air se développe graduellement (CHANSON 1989, 1992a).

L'entraînement d'air dans des écoulements supercritiques est un paramètre important, caractérisant l'augmentation de volume de l'écoulement (FALVEY 1980). De plus, la présence d'air dans les écoulements à grandes vitesses permet de réduire ou d'empêcher les dommages dus à la cavitation (MAY 1987, CHANSON 1989). Mais aussi, WOOD (1983) a montré que le coefficient de perte de charge des écoulements à surface libre aérés décroît avec la quantité d'air entraînée.

Le présent document continue une étude détaillée des écoulements à surface libre avec aération (CHANSON $1989,1992 a)$. Le problème de la réduction du coefficient de perte de charge est discuté, et une comparaison de mesures expérimentales obtenues sur modèles et prototypes de coursiers est présentée. On propose une explication du mécanisme de réduction des pertes de charge. Puis, on développe une analogie avec la réduction de traînée observée avec des écoulements de polymères dilués, et lors de l'injection de micro-bulles d'air dans des couches limites turbulentes.

Drag reduction in open channel flows with air entrainment.

Augmentation of the maximum discharge capacity of a spillway

In supercritical open channel flows air is entrained at the free surface. Such air-water flows, called self-aerated flows, exhibit smaller friction losses than non-aerated flows. New data on drag reduction in self-aerated flows are presented. It is shown that the drag reduction process is linked with the presence of an air concentration boundary layer next to the channel bottom. An analogy with dilute polymer solutions and micro bubble modified boundary layers is developed and it is suggested that the presence of air next to the bottom increases the effective viscosity of the mixture and the sublayer thickness $\star$. The process of drag reduction could be used to increase the maximum discharge capacity of a spillway. Combining self-aeration and the use of bottom aeration devices, the friction factor along the spillway can be reduced, inducing an increase of maximum discharge. 
1. Entraînement d'air au long d'un coursier d'évacuateur de crues.

Air entrainment along a spillway.

2. Profils de concentrations en air et de vitesses à Aviemore CaIN (1978).

Air concentration and velocity distributions at Aviemore spillway - CAIN (1978).

3. Concentrations en air dans la couche limite de concentration en air.

Air concentration distribution in the air concentration boundary layer.

4. Epaisseur de couche limite de concentration en air $\Delta_{a b}$ en fonction du nombre de Reynolds $\mathrm{Re}+$ et de la concentration en air $C_{b}$.

Thickness of the air concentration boundary layer as a function of the Reynolds number.

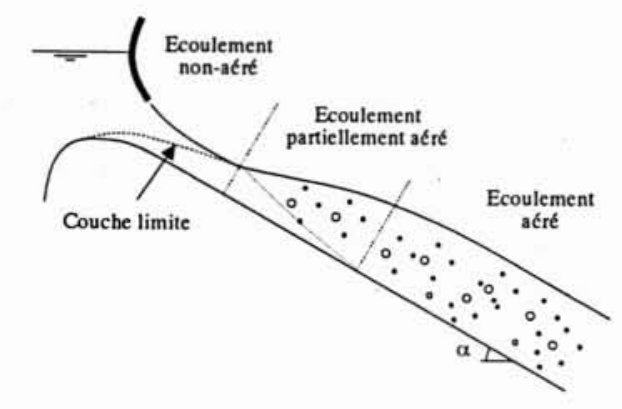

1.

2.
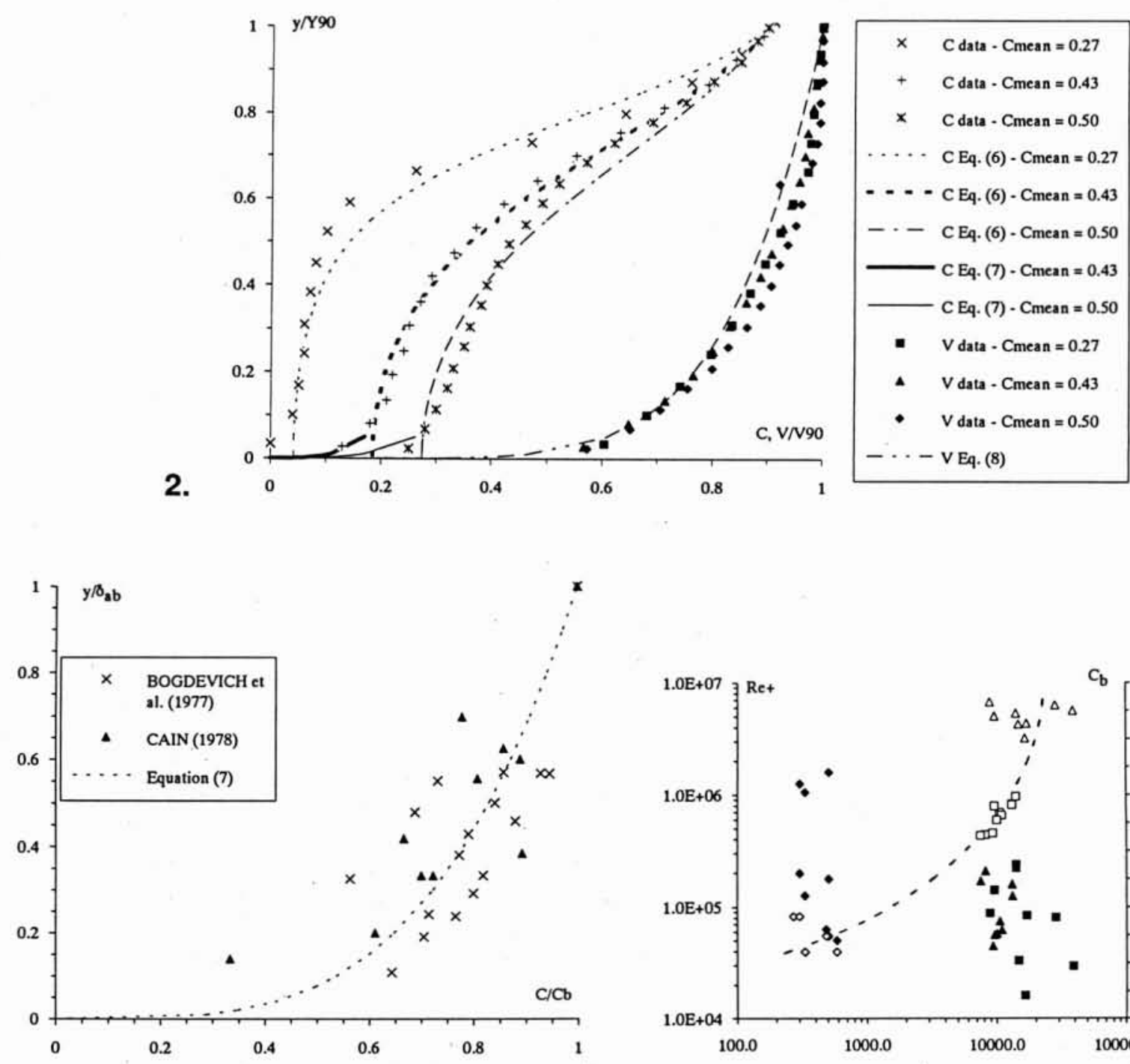

3.

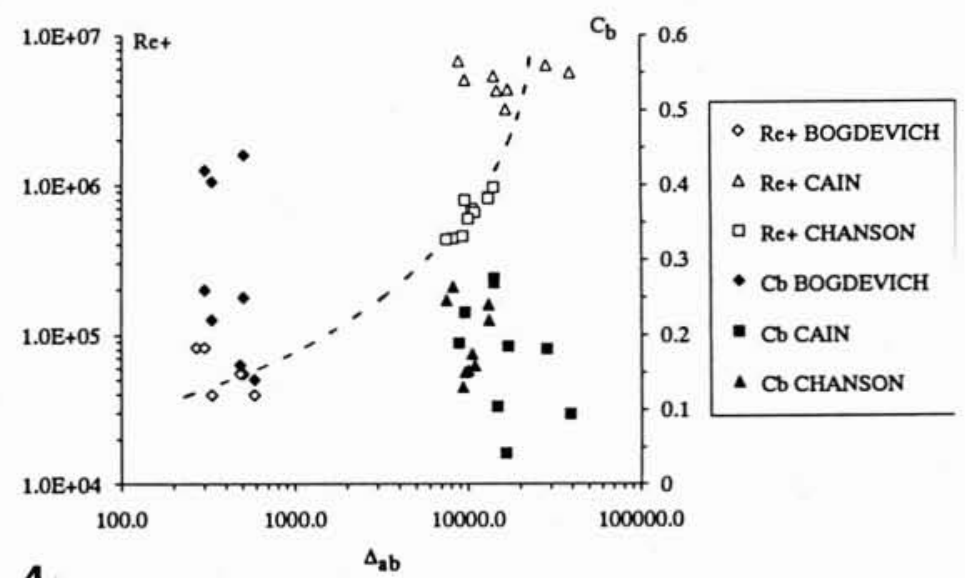

4. 


\section{Mécanismes d'entraînement d'air}

Pour des écoulements à grandes vitesses, les vitesses de fluctuations turbulentes, près de la surface libre, induisent un processus d'aération. A travers la surface libre, l'air s'engouffre dans l'écoulement, sous forme de bulles d'air et de poches d'air (CAIN 1978, Wood 1991). Le phénomène d'aération apparaît lorsque le niveau de turbulence est suffisant pour compenser les forces de tension superficielles et la poussée d'Archimède. C'est-à-dire, lorsque la fluctuation de la composante de la vitesse, perpendiculaire à la surface libre, $v^{\prime}$ est supérieure à la composante de la vitesse limite d'ascension des bulles d'air et à la résistance de la tension superficielle (SENE 1984, ERVINE and FALVEY 1987). Ces conditions s'écrivent :

$$
v^{\prime}>u_{r} * \cos \alpha
$$

et

$$
v^{\prime}>\sqrt{\frac{8 * \sigma}{\rho_{w} * d_{b}}}
$$

où $u_{r}$ est la vitesse limite d'ascension de la bulle d'air, $\alpha$ est la pente du coursier, $\sigma$ est la tension superficielle, $\rho_{w}$ est la masse volumique de l'eau, et $d_{b}$ est le diamètre de la bulle d'air. En faisant l'hypothèse d'une vitesse limite d'ascension $u_{r}$ de l'ordre de $0,25 \mathrm{~m} / \mathrm{s}$, le système d'équations (1) et (2) indique que le processus d'entraînement d'air commence pour des vitesses turbulentes $v^{\prime}$ de l'ordre de 0,1 à $0,3 \mathrm{~m} / \mathrm{s}$. Sur des coursiers d'évacuateurs de crues, les écoulements sont hydrauliquement rugueux, et les fluctuations de vitesses satisfont le critère d'entraînement d'air (équations (1) et (2)), en aval de la position où la couche limite turbulente atteint la surface libre (fig. 1).

\section{Définitions}

La concentration en air $C$ est définie comme le volume d'air par unité de volume. La profondeur de référence, pour un écoulement aéré, est :

$$
d=\int_{0}^{Y_{90}}(1-C) * \mathrm{~d} y
$$

où $y$ est mesuré perpendiculairement à la surface du coursier et $Y_{90}$ est la distance depuis la surface du coursier, où la concentration en air atteint $90 \%$. La concentration en air moyenne dans une section, $C_{\text {mean }}$, est définie comme :

$$
\left(1-C_{\text {mean }}\right) * Y_{90}=d .
$$

La vitesse moyenne de l'écoulement $U_{w}$ est :

$$
U_{w}=\frac{q_{w}}{d}
$$

où $q_{w}$ est le débit par unité de largeur. A la distance $y=Y_{90}$ depuis la surface du coursier, la vitesse est égale à $V_{90}$.

\section{Profils de concentrations en air et de vitesses}

Dans un écoulement à surface libre avec entraînement d'air, le profil de concentration en air peut être calculé en utilisant le modèle de diffusion des bulles d'air, développé par WOOD (1984) :

$$
C=\frac{B^{\prime}}{B^{\prime}+\exp \left(-G^{\prime} * \cos \alpha * y^{\prime 2}\right)}
$$

où $B^{\prime}$ et $G^{\prime}$ sont fonctions uniquement de la concentration en air moyenne, et $y^{\prime}=y / Y_{90}$. Le tableau $I$, en annexe, indique les valeurs de $B^{\prime}$ et $G^{\prime}$ (colonnes 2 et 3 ), calculées à partir des données expérimentales de STRAUB and ANDERSON (1958), en fonction de la concentration en air moyenne (colonne 1).

Près de la surface du coursier, cependant, les mesures de CAIN (1978) et CHANSON (1988), obtenues sur prototype et modèle, divergent de l'équation (6), et impliquent que la concentration en air tend vers zéro à la paroi solide $(C(y=0)=0)$ (fig. 2). La présence d'une couche limite de concentration en air, sur un coursier, a été initialement mise en évidence par l'auteur (CHANSON 1989), et est en accord avec les résultats obtenus par BOGDEVICH et al. (1977), MADAVAN et al. (1984) et MARIE et al. (1991), qui ont étudié la diffusion de micro-bulles d'air dans une couche limite turbulente $(t a b l . I I)$. Leurs mesures indiquent, aussi, que la concentration de bulles d'air tend vers zéro à la paroi solide. En réexaminant les données expérimentales de Bogdevich et al. (1977) et CAIN (1978), on déduit que le profil de concentrations en air, dans la couche limite de concentration en air, s'exprime :

$$
C=C_{b} *\left(\frac{y}{\delta_{a b}}\right)^{0.270}
$$

où $\delta_{a b}$ est l'épaisseur de couche limite de concentration en air, et $C_{b}$ est la concentration en air à la limite supérieure de la couche limite de concentration en air. $C_{b}$ satisfait la continuité entre les équations (6) et (7). La figure 3 montre une comparaison entre les mesures expérimentales et l'équation (7). Pour des écoulements sur un coursier d'évacuateur de crues, la profondeur d'eau est largement supérieure à l'épaisseur $\delta_{a b}\left(\delta_{a b} \ll Y_{90}\right)$, et $C_{b}$ est égale à : $C_{b}=B^{\prime} /\left(B^{\prime}+1\right)($ tabl. $I$, colonne 4$)$.

Pour les expérimentations de Bogdevich et al. (1977), CAIN (1978) et Chanson (1988), l'auteur a recalculé l'épaisseur de la couche limite des bulles d'air. Les résultats sont regroupés sur la figure 4, présentant l'épaisseur adimensionnelle de la couche limite de concentration en air, $\Delta_{a b}$, en fonction des paramètres caractéristiques de la couche limite: le nombre de Reynolds $\operatorname{Re}+=V_{0} * Y_{0} / \nu_{w^{\prime}}$ et la concentration en air $C_{b}$. Les notations suivantes sont utilisées: $\Delta_{a b}=$ $\delta_{a b} * V * / \nu_{w}, V *$ est la vitesse de frottement, $\nu_{w}$ est la viscosité cinématique de l'eau, $V_{0}$ est la vitesse à limite supérieure de la couche limite turbulente et $Y_{0}$ est l'épaisseur de la couche limite turbulente. Pour les écoulements à surface libre avec aération (fig. 1), la couche limite turbulente est complètement développée, et satisfait $V_{0}=V_{90}$ et $Y_{0}=Y_{90}$. 


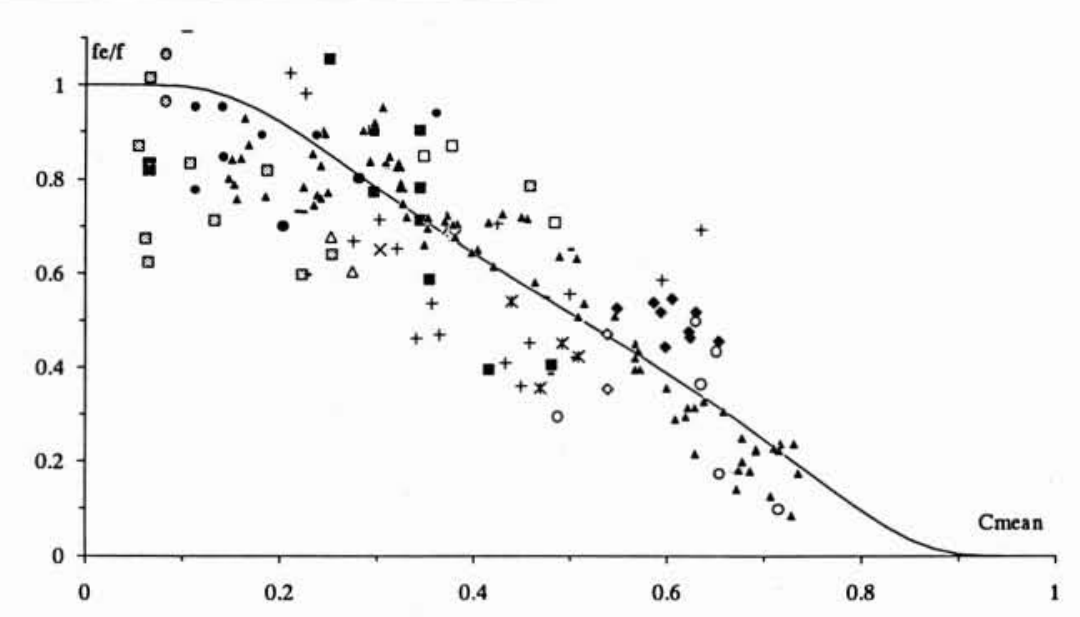

\begin{tabular}{|c|c|c|c|c|c|c|c|c|c|}
\hline व & Ak-Tepe & $\circ$ & Bencok & - & Big Hill & a & Boise & $\Delta$ & Dago \\
\hline$x$ & Erevan & $x$ & Gizel'don & + & Hat Creek & - & Kittitas & $\Delta$ & Mallnitz \\
\hline - & $\begin{array}{l}\text { Mostarsko } \\
\text { Blato }\end{array}$ & $\bullet$ & Rapid Flume & o & Rutz & $\circ$ & Spring Gully & $\cdot$ & IWP \\
\hline 4 & $\begin{array}{l}\text { St Anthony } \\
\text { Falls }\end{array}$ & $\bullet$ & Vienne & 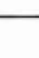 & Eq. (9) & & & & \\
\hline
\end{tabular}

5. Réduction du coefficient de frottement sur modèles et prototypes.
Drag reduction observed on prototype spillways and spillway models.
La figure 4 montre : 1) que l'épaisseur de la couche limite de concentration en air $\Delta_{a b}$ augmente avec le nombre de Reynolds $\operatorname{Re}+$, et semble tendre vers une valeur limite, et 2) que l'épaisseur de la couche limite de concentration en air varie peu en fonction de la concentration en air $C_{b}$. En première approximation, $\Delta_{a b}$ est indépendante de la concentration en air. On note que l'équation (7) a été obtenue pour $0,04<C_{b}<0,70$, et qu'elle peut être considérée comme indépendante de la concentration en air à la limite supérieure de la couche limite de bulles d'air.

Dans les écoulements à surface libre, la présence d'air affecte peu le profil des vitesses. CAIN et WoOD (1981) ont montré que la distribution des vitesses suit une loi de puissance :

$$
\frac{V}{V_{90}}=\left(\frac{y}{Y_{90}}\right)^{1 / n}
$$

où $V$ est la vitesse à une distance $y$ de la surface du coursier. Pour le coursier de l'évacuateur de crues du barrage d'Aviemore, et pour le modèle de l'évacuateur de crues du barrage de Clyde, les mesures de profils de vitesses indiquent que l'exposant est égal à : $n=6,0$ (Chanson 1989). Ce résultat, ayant été obtenu avec des concentrations en air moyennes comprises entre 0 et 0,50 , est en première approximation indépendant de la concentration en air moyenne. L'auteur (CHANSON 1992a) a démontré que la vitesse $V_{90}$ se déduit de l'équation de conservation de la masse, en combinant les équations (6), (7) et (8).

\section{Réduction du coefficient de perte de charge}

\section{Présentation}

Bien que la présence d'air dans les écoulements à surface libre n'affecte pas les profils de vitesses, JEVDJEVICH et LEVIN (1953) et KILLEN (1968) ont suggéré que la présence de bulles d'air modifie la couche limite turbulente et induit une réduction des contraintes de cisaillement et du coefficient de perte de charge. En utilisant des mesures obtenues avec des écoulements uniformes, WoOD (1983) a appliqué l'équation de conservation de l'énergie à des écoulements aérés. Son analyse a montré que le coefficient de perte de charge diminue lorsque la concentration en air moyenne augmente.

Cette conclusion a été vérifiée avec des données expérimentales obtenues sur des modèles et prototypes de coursiers d'évacuateurs de crues, ainsi que pour des écoulements sur des canaux avec enrochements (CHANSON 1992a).

\section{Application aux coursiers d'évacuateurs de crues}

En utilisant la méthode développée par WoOD (1983, 1991) et étendue par CHANSON (1992a), l'auteur a réanalysé de nouvelles mesures expérimentales, obtenues sur des coursiers de formes diverses en Australie, Autriche, Indonésie, Etats-Unis, Union Soviétique et Yougoslavie (tabl. III). Pour l'ensemble de ces données (modèles et prototypes), on peut expliciter la réduction du coefficient de perte de charge par la formule suivante : 


$$
\frac{f_{e}}{f}=0,5 *\left(1+\tanh \left(0,628 * \frac{0,514-C_{\text {mean }}}{C_{\text {mean }} *\left(1-C_{\text {mean }}\right)}\right)\right)
$$

où $f$ est le coefficient de perte de charge de l'écoulement non aéré, $f_{e}$ est le coefficient de perte de charge de l'écoulement avec entraînement d'air, et tanh est la fonction tangente hyperbolique. Pour l'ensemble de ces données expérimentales, la rugosité relative $k_{s} / D_{H}$ est comprise entre 0,0003 et 0,04 , et le nombre de Reynolds $\operatorname{Re}=U_{w} * D_{H} / \nu_{w}$ est compris entre 50.000 et $3.10^{7}$.

Sur la figure 5 , on a comparé l'équation (9) avec les résultats expérimentaux. La figure 5 montre que le coefficient de perte de charge décroît lorsque la concentration en air moyenne est supérieure à $20 \%$. Pour $C_{\text {mean }}>0,20$, la concentration en air près de la surface du coursier devient non nulle (WOOD 1983, KoschiTZKY et KoBUs 1988), et des bulles d'air interagissent avec les mouvements tourbillonnaires près de la paroi.

\section{Discussion}

Un aspect important du processus de réduction de coefficient de perte de charge est le lien entre les profils de vitesses, et de concentrations en air près de la paroi. L'auteur suggère une analogie entre ce processus et les observations faites avec des solutions diluées de polymères (VIRK 1975, LUMLEY 1977) et lors de l'injection de micro-bulles d'air dans une couche limite turbulente (BOGDEVICH et al. 1977, MARIE et al. 1991). L'injection de polymères ou de micro-bulles d'air dans une couche limite turbulente entraîne une réduction importante du coefficient de perte de charge.

Une explication du mécanisme de réduction des pertes de charge est basée sur l'hypothèse que la couche limite de concentration en air joue un rôle similaire à la souscouche visqueuse et sous-couche élastique : la présence de bulles d'air, près de la paroi solide, augmente la viscosité dynamique du fluide, et induit un épaississement de la sous-couche visqueuse (LUMLEY 1977, MARIE 1987). MARIE (1987) a développé une solution analytique pour prédire la réduction du coefficient de traînée, résultant de la présence de bulles d'air près de la paroi. Ce modèle se base sur une augmentation de l'épaisseur de la sous-couche visqueuse, due à l'accroissement de la viscosité cinématique du fluide près de la paroi solide. La densité de l'air est 100 fois plus petite que celle de l'eau, et la masse volumique du fluide près de la paroi est égale à :

$$
\rho_{a w}=\rho_{w} *\left(1-C_{b}\right)
$$

où $\rho_{a w}$ est la masse volumique du mélange eau-air, et $C_{b}$ est la concentration en air à la limite supérieure de la couche limite de concentration en air. EINSTEIN (1906, 1911) a démontré que la viscosité dynamique du mélange diphasique est égale à :

$$
\mu_{a w}=\mu_{w} *\left(1+2.5 * C_{b}\right)
$$

où $\mu_{a w}$ est la viscosité du mélange eau-air et $\mu_{w}$ est la viscosité dynamique de l'eau. En combinant les équations (10) et (11), les calculs de MARIE (1987) se réduisent à :

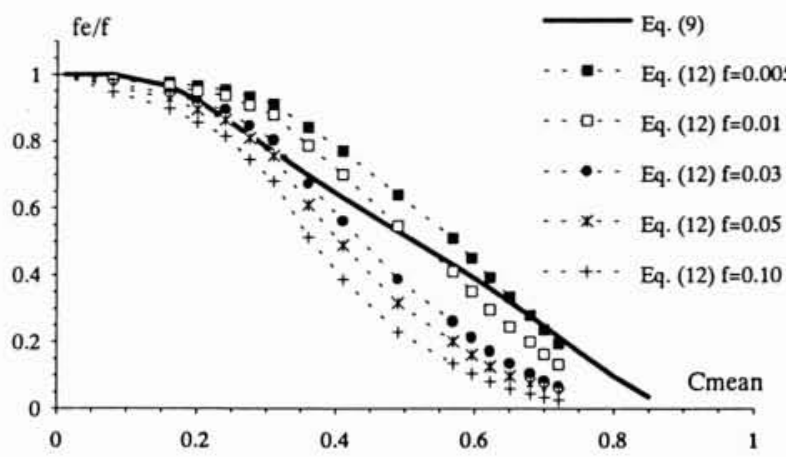

6. Comparaisons entre les équations (9) et (12).

Drag reduction : comparison between equations (9) and (12).

$$
\begin{aligned}
\frac{f_{c}}{f}=[1+ & \sqrt{\frac{f}{8}} *\left(10.5 *\left(\frac{1+2,5 * C_{b}}{1-C_{b}}-1\right)-\right. \\
& \left.\left.-2.44 * \operatorname{Ln}\left(\frac{1+2,5 * C_{b}}{1-C_{b}}\right)\right)\right]^{-9 / 5} .
\end{aligned}
$$

La figure 6 présente $f$ df calculé avec l'équation (12) en fonction de la concentration en air moyenne, en utilisant la concentration en air $C_{b}$ déduite de la table I (colonne 4), pour plusieurs valeurs du coefficient de perte de charge $f$. Pour un coursier en béton lisse, $f=0.03$ est une valeur typique.

Dans la couche limite de concentration en air, les mesures (CAIN 1978, Chanson 1988, 1992b) et les calculs (CHANSON 1992c) indiquent que les bulles d'air sont de petites tailles $\left(d_{b}<1 \mathrm{~mm}\right)$ près de la surface du coursier. Ces valeurs sont du même ordre de grandeur que les mesures obtenues par BoGDEvicH et al. (1977) et MADAVAN et al. (1984, 1985) (tabl. II), dont les résultats ont été utilisés pour valider le modèle de MARIE (1987). En conséquence, il est cohérent de comparer les équations (9) et (12) (fig. 6). Ces deux équations donnent des résultats très proches, et ce résultat confirme l'hypothèse d'une analogie entre les mécanismes de réduction du coefficient de perte de charge, pour les écoulements à surface libre avec entraînement d'air, et lors de l'injection de bulles d'air dans des couches limites turbulentes.

L'auteur (CHANSON 1992b) a suggéré que les bulles d'air de petites tailles, observées dans la couche limite de concentration en air, se comportent comme des sphères rigides (COMOLET 1979), offrant une résistance importante aux contraintes de cisaillement tendant à rompre les bulles d'air. De telles bulles d'air rigides se comportent comme des macro-molécules de polymères, et bloquent la génération de bouffées turbulentes près de la paroi, de manière analogue au comportement des macro-molécules de polymères dans la sous-couche élastique (VIRK 1975). Les molécules de polymères ont des propriétés spécifiques d'élongation qui induisent un accroissement de la viscosité dans la sous-couche élastique, définie comme la région comprise entre la sous-couche visqueuse et la zone 


\section{H. CHANSON}

de recouvrement (zone de lois logarithmiques de vitesses) (VIRK 1975, TAM et al. 1992). Pour des écoulements aérés, la présence de bulles d'air, à la limite supérieure de la couche limite de concentration en air, est similaire à la présence de macro-molécules étirées dans la sous-couche élastique. Les bulles d'air et les macro-molécules étirées de polymères induisent un accroissement de la viscosité, et « cette augmentation de la viscosité dans les couches turbulentes, et pas dans la sous-couche visqueuse, supprime les tourbillons transportant les contraintes de Reynolds dans la zone de recouvrement, induisant un épaississement de la sous-couche visqueuse, et une réduction de la traînée " (LUMLEY 1977).

\section{Application}

Le phénomène de réduction du coefficient de frottement doit être pris en compte lors de la conception d'un coursier d'évacuateur de crues. Pour une hauteur de chute donnée, la figure 5 montre une réduction du coefficient de perte de charge, et donc un accroissement de la vitesse moyenne. Cette augmentation de la vitesse de l'écoulement est un paramètre important pour le calcul d'un bassin de dissipation en aval d'un coursier, ou pour le positionnement d'une fosse en aval d'un tremplin («saut de ski »).

De plus, il serait envisageable d'accroître la capacité d'un coursier par une augmentation du débit maximal, résultant d'une réduction des pertes de charge. Récemment, des aménagements de barrages ont été soumis à des débits de crues largement supérieurs aux débits maximaux des coursiers d'évacuateurs de crues ( $t a b l . I V)$. Bien que, dans deux cas, les barrages aient été rompus à cause de problèmes d'ouvertures de vannes de réglage, ces événements hydrologiques soulignent l'intérêt que pourrait apporter une augmentation de la capacité des coursiers.

Pour des aménagements de moyenne et hautes chutes, le débit maximal qu'un évacuateur de crues peut écouler, est égal au produit de la vitesse maximale par la hauteur d'eau : $q_{\max }=V_{\max } * d$. La hauteur d'eau maximale est déterminée par la hauteur des bajoyers, et la vitesse maximale est déduite de la formule de Chézy. Pour un coursier de grande largeur, la vitesse maximale d'un écoulement uniforme avec entraînement d'air est :

$$
\left(V_{\max }\right)_{a w}=\sqrt[3]{\frac{8 * g}{f} * q_{w} * \sin \alpha *} \quad \sqrt[3]{\frac{f}{f_{e}}} .
$$

Considérant un écoulement uniforme, pour une même hauteur d'eau $d$, l'augmentation du débit maximal, résultant de l'aération de l'écoulement, peut être exprimée :

$$
\frac{\left(q_{\max }\right)_{a w}}{q_{\max }}=\sqrt{\frac{f}{f_{e}}}
$$

où $q_{\max }$ est le débit maximal sans entraînement d'air et $\left(q_{\max }\right)_{a w}$ est le débit maximal avec aération de l'écoulement. L'équation (14) prédit l'augmentation de débit résultant de l'aération de l'écoulement pour une hauteur d'eau identique $d$, mais elle ne prend pas en compte l'augmentation du volume réel d'un écoulement aéré.

\section{Utilisation d'aérateurs de fond}

Si l'entraînement d'air à la surface libre est insuffisant pour augmenter la débitance d'un coursier, une quantité d'air additionnelle peut être introduite artificiellement à l'aide de dispositifs de ventilation, appelés aérateurs. Les aérateurs de fond permettent d'introduire de grandes quantités d'air sur de faibles distances, et ils ont été initialement développés pour réduire les dommages dus à la cavitation (QUINTELA 1980, VISCHER et al. 1982). En aval d'un aérateur, l'écoulement aéré se comporte comme un écoulement à surface libre avec entraînement d'air (CHANSON 1989, HAGer 1992). En se basant sur la méthode développée par l'auteur pour calculer les caractéristiques de l'écoulement en aval d'un aérateur, on peut prédire la localisation optimale du (des) aérateur(s).

Le premier aérateur doit être positionné le plus près possible de l'entrée du coursier, afin d'aérer rapidement l'écoulement. En aval de cet aérateur, la quantité d'air entraînée augmente pour des coursiers raides $\left(\alpha>20^{\circ}\right)$, et décroît pour des radiers à faibles pentes (CHANSON 1989). Dans tous les cas, il est nécessaire de calculer les caractéristiques de l'écoulement en aval de l'aérateur. Si la quantité d'air, requise pour accroître la débitance du coursier, n'est pas obtenue, un ou plusieurs aérateurs supplémentaires doivent être installés.

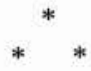

\section{Conclusion}

Les caractéristiques des écoulements à surface libre, avec entraînement d'air, ont été résumées. Bien que la distribution des vitesses soit peu affectée par l'entraînement d'air, les résultats expérimentaux obtenus sur modèles et prototypes montrent une réduction du coefficient de perte de charge avec la quantité d'air entraînée (fig. 5). Le profil de concentration en air est caractérisé par la présence d'une couche limite de concentration en air près de la surface du coursier. Par analogie avec les solutions diluées de polymères, et les écoulements avec injection de bulles d'air dans une couche limite turbulente, on a montré que la couche limite de concentration en air a un rôle prédominant dans le mécanisme de réduction du coefficient de frottement. La présence de bulles d'air près de la paroi accroît la viscosité effective du fluide, et induit un épaississement de la sous-couche visqueuse et une réduction de la trainée.

Ce phénomène peut être appliqué pour accroître la vitesse maximale et le débit maximal sur un coursier. On pourrait utiliser des aérateurs de fond, régulièrement espacés, pour induire artificiellement un entraînement d'air, réduire le coefficient de perte de charge et accroître la vitesse maximale sur le radier d'un coursier. 
ANNEXE 1

Tables de résultats

Table I - Paramètres des profils de concentrations en air et de vitesses pour des écoulements à surface libre aérés

Table I - Air concentration and velocity distribution parameters in self-aerated flows

\begin{tabular}{|c|c|c|c|c|}
\hline $\begin{array}{c}\mathrm{C}_{\text {mean }} \\
\text { (1) } \\
\end{array}$ & $\begin{array}{c}G^{\prime *} \cos \alpha \\
\text { (a) } \\
\text { (2) }\end{array}$ & $\begin{array}{l}\mathrm{B}^{\prime} \\
\text { (a) } \\
\text { (3) } \\
\end{array}$ & $\begin{array}{l}C_{b} \\
(b) \\
(4)\end{array}$ & $\begin{array}{c}f_{e} / f \\
\text { Eq. (9) } \\
(5) \\
\end{array}$ \\
\hline 0,0 & + infini & 0,00 & 0,00 & 1,0 \\
\hline 0,161 & 7,999 & 0,003021 & 0,02 & 0,964 \\
\hline 0,241 & 5,744 & 0,028798 & 0,04 & 0,867 \\
\hline 0,310 & 4,834 & 0,07157 & 0,07 & 0,768 \\
\hline 0,410 & 3,825 & 0,19635 & 0,17 & 0,632 \\
\hline 0,569 & 2,675 & 0,62026 & 0,36 & 0,430 \\
\hline 0,622 & $2, .401$ & 0,8157 & 0,46 & 0,360 \\
\hline 0,680 & $1, .8942$ & 1,3539 & 0,55 & 0,277 \\
\hline 0,721 & 1,5744 & 1,8641 & 0,64 & 0,215 \\
\hline
\end{tabular}

Notes : (a) calculé à partir des mesures de STRAUB and ANDERSON (1958)

(b) déduit de l'équation (6): $C_{b}=B^{\prime} /\left(B^{\prime}+1\right)$

Table II - Mesures expérimentales de couches limites de concentrations en air Table II - Drag reduction experiments and air concentration boundary layer characteristics

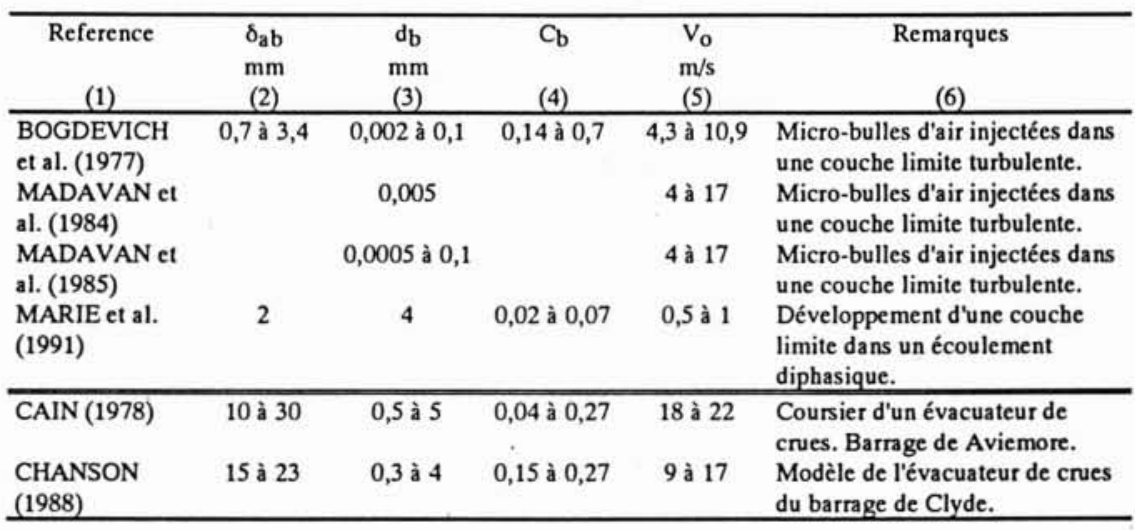

Notation: $\delta_{\mathrm{ab}} \quad$ épaisseur de la couche limite de concentration en air

$d_{b}$ diamètre moyen des bulles d'air

$C_{b}$ concentration en air à $y=\delta_{a b}$

$\mathrm{V}_{\mathrm{o}} \quad$ vitesse à la limite supérieure de couche limite turbulente 


\section{H. CHANSON}

(annexe 1 - suite)

Table III - Ecoulements à surface libre avec entraînement d'air : modèles et prototypes

Table III - Self-aerated flow measurements on prototype and model spillways

\begin{tabular}{|c|c|c|c|c|}
\hline $\begin{array}{l}\text { Coursier } \\
\text { (1) }\end{array}$ & $\begin{array}{l}\text { Pente } \\
\text { degrés } \\
\text { (2) }\end{array}$ & $\begin{array}{l}9 w \\
\mathrm{~m}^{2} / \mathrm{s} \\
(3)\end{array}$ & $\begin{array}{l}\text { Réf. } \\
\text { (4) }\end{array}$ & $\begin{array}{c}\text { Remarques } \\
\text { (5) }\end{array}$ \\
\hline \multicolumn{5}{|l|}{ Prototypes } \\
\hline Ak-Tepe, URSS & 21,8 & 2,3 à 8,0 & [A] & Béton rugueux. $\mathrm{W}=5 \mathrm{~m} \cdot \mathrm{k}_{\mathrm{s}}=5 \mathrm{~mm}$. \\
\hline $\begin{array}{l}\text { Aviemore, Nouvelle } \\
\text { Zélande }\end{array}$ & 45,0 & 2,23 et 3,16 & [CA] & Béton. $\mathrm{k}_{\mathrm{S}}=1 \mathrm{~mm}$. \\
\hline Bencok, Indonésie & 31,05 & 2,9 à 6,0 & {$[\mathrm{~A}, \mathrm{E}, \mathrm{L}]$} & $\mathrm{W}=1 \mathrm{~m} \cdot \mathrm{ks}_{\mathrm{s}}=2 \mathrm{~mm}$. \\
\hline Big Hill, Australie & 4,2 & 0,74 à 0,82 & [M] & Béton lisse. \\
\hline Boise, USA & 4,6 à 12,2 & 0,01 à 0,8 & [S] & Béton. $\mathrm{W}=0,9$ à $1,8 \mathrm{~m} \cdot \mathrm{k}_{\mathrm{s}}=1 \mathrm{~mm}$. \\
\hline Dago, Indonésie & 13,8 & 0,74 à 1,39 & {$[\mathrm{~A}, \mathrm{E}, \mathrm{L}]$} & $\mathrm{W}=1 \mathrm{~m} \cdot \mathrm{k}_{\mathrm{s}}=1 \mathrm{~mm}$ \\
\hline Erevan, URSS & 21,8 & 0,38 à 1,55 & [A] & $\begin{array}{l}\text { Basalte rugueux avec un mortier de ciment. } \\
W=4 \mathrm{~m} . \mathrm{k}_{\mathrm{S}}=10 \mathrm{~mm} .\end{array}$ \\
\hline Gizel'don, URSS & 28,1 & 0,49 à 1,28 & [A] & $\begin{array}{l}\text { Canal en planches rabotées. } \mathrm{W}=6 \mathrm{~m} \cdot \mathrm{k}_{\mathrm{s}}= \\
0,3 \mathrm{~mm} .\end{array}$ \\
\hline Hat Creek, USA & $\begin{array}{c}23,45 \text { à } \\
34,75\end{array}$ & 1,86 à 6,4 & {$[\mathrm{H}]$} & Béton rugueux. $\mathrm{W}=1,75 \mathrm{~m} . \mathrm{k}_{\mathrm{s}}=5 \mathrm{~mm}$ \\
\hline Kittitas, USA & 33,2 & 2,24 à 11,7 & {$[\mathrm{H}]$} & Béton érodé. $\mathrm{W}=2,44 \mathrm{~m} . \mathrm{k}_{\mathrm{s}}=10 \mathrm{~mm}$. \\
\hline Mallnitz, Autriche & 22,2 & & {$[A, E, L]$} & Béton. $\mathrm{W}=2 \mathrm{~m}$. \\
\hline $\begin{array}{l}\text { Mostarsko Blato, } \\
\text { Yougoslavie }\end{array}$ & & 0,71 à 3,44 & [ग] & Pierre de taille. $W=5,35 \mathrm{~m} \cdot \mathrm{k}_{\mathrm{s}}=20 \mathrm{~mm}$. \\
\hline Rapid Flume, USA & 20,1 & 1,76 & [H] & $\begin{array}{l}\text { Canal en planches rabotées paintes. } \\
\mathrm{W}=1,4 \mathrm{~m} \cdot \mathrm{k}_{\mathrm{S}}=0,1 \mathrm{~mm} \text {. }\end{array}$ \\
\hline Spring Gully, Australie & 5,3 & & [M] & $\begin{array}{l}\text { Béton lisse. Canal semi-circulaire de rayon } \\
R=0,61 \mathrm{~m} \text {. }\end{array}$ \\
\hline Rutz, Autriche & 34 & 0,4 à 2 & [I] & Béton. Canal trapezoïdal. \\
\hline \multicolumn{5}{|l|}{ Modèles } \\
\hline $\begin{array}{l}\text { Modèle de Clyde, } \\
\text { Nouvelle Zélande }\end{array}$ & 52,3 & 0,21 à 0,48 & {$[\mathrm{CH}]$} & $\begin{array}{l}\text { Perspex. En aval d'un aérateur. } W=0,25 \mathrm{~m} \text {. } \\
\mathrm{k}_{\mathrm{s}}=0,1 \mathrm{~mm} \text {. }\end{array}$ \\
\hline IWP, URSS & 16,7 et 29,7 & 0,064 à 0,13 & [A] & $\begin{array}{l}\text { Canal en planches rabotées paintes. } \\
W=0,25 \mathrm{~m} \cdot \mathrm{k}_{\mathrm{S}}=0,1 \mathrm{~mm} \text {. }\end{array}$ \\
\hline Lab. de Vienne, Autriche & 8,7 à 31,2 & 0,04 à 0,178 & {$[\mathrm{~A}, \mathrm{E}, \mathrm{J}, \mathrm{L}]$} & Canal en planches rabotées. $W=0,25 \mathrm{~m}$. \\
\hline St Anthony Falls, USA & 7,5 à 75 & 0,14 à 0,93 & {$[\mathrm{SA}]$} & $\begin{array}{l}\text { Rugosités artificielles : } \mathrm{k}_{\mathrm{s}}=0,7 \mathrm{~mm} . \mathrm{W}=0,46 \\
\mathrm{~m} \text {. }\end{array}$ \\
\hline \multicolumn{5}{|l|}{ Canal en enrochement } \\
\hline Obernach, Allemagne & 6 à 34 & & {$[\mathrm{HS}]$} & $\mathrm{k}_{\mathrm{S}}=0,1 \mathrm{à} 0,35 \mathrm{~m}$. \\
\hline
\end{tabular}

Notation : $\quad q_{w} \quad$ discharge par unité de largeur

$\mathrm{k}_{\mathrm{S}} \quad$ rugosité uniforme équivalente

W largeur du coursier

Références: [A] AIVAZYAN (1986); [CA] CAIN (1978); [CH] CHANSON (1988); [E] EHRENBERGER (1926); [H] HALL (1943); [HS] HARTUNG et SCHEUERLEIN (1970); [I] INNEREBNER (1924); J] JEVDJEVICH et LEVIN (1953); [L] LEVIN (1955); [M] MICHELS et LOVELY (1953); [S] STEWART (1913); [SA] STRAUB et ANDERSON (1958).

Table IV - Exemples de débits de crues exceptionnelles sur des évacuateurs de crues de surface Table IV - Examples of peak flood flows in open channel spillways

\begin{tabular}{|c|c|c|c|c|c|}
\hline Ouvrage & Pays & $\begin{array}{l}\text { Année de crue } \\
\text { catastrophique }\end{array}$ & Débit de crue & $\begin{array}{c}\text { Débit } \\
\text { maximal du } \\
\text { coursier } \\
\mathrm{m}^{3} / \mathrm{s} \\
(5)\end{array}$ & Commentaires \\
\hline $\begin{array}{l}\text { Barrage de } \\
\text { Palagnedra }\end{array}$ & Suisse & 1978 & 3000 à 3500 & $450\left(^{*}\right)$ & $\begin{array}{l}\text { Submersion par } \\
\text { déversement du barrage }\end{array}$ \\
\hline Barrage de Tous & Espagne & 1982 & 16000 & 7000 & Destruction du barrage \\
\hline Barrage de Belci & Roumanic & 1991 & 2200 & 1400 & Destruction du barrage \\
\hline
\end{tabular}

Note

(*) Le coursier pouvait écouler exceptionnellement jusqu'd $900 \mathrm{~m}^{3} / \mathrm{s}$. Lors de la submersion par déversement du barrage, il est estimé que le coursier évacuait $2000 \mathrm{~m}^{3} / \mathrm{s}$. 


\section{ANNEXE 2}

Références

AIVAZYAN, O.M. (1986). "Stabilized Aeration on Chutes." Gidrotekhnicheskoe Stroitel'stvo, No. 12, pp. 33-40 (Hydrotechnical Construction, 1987, Plenum Publ., pp. 713-722) (en Anglais).

BOGDEVICH, V.G., EVSEEV, A.R., MLYUGA, A.G., et MIGIRENKO, G.S. (1977). "Gas-Saturation Effect on Near-Wall Turbulence Characteristics." Proc. of the 2nd Intl. Conf. on Drag Reduction, BHRA Fluid Eng., Cambridge, UK, Paper D2, pp. 25-37 (en Anglais).

CAIN, P. (1978). "Measurements within Self-Aerated Flow on a Large Spillway." Ph.D. Thesis, Ref. 7818, 1978, Univ. of Canterbury, Christchurch, New Zealand (en Anglais).

CAIN, P., et WOOD, I.R. (1981). "Measurements of Self-aerated Flow on a Spillway." Jl. Hyd. Div., ASCE, 107, HY11, pp. 1425-1444 (en Anglais).

CHANSON, H. (1988). "A Study of Air Entrainment and Aeration Devices on a Spillway Model." Research Rep. 88-8, Oct., Univ. of Canterbury, New Zealand (en Anglais).

CHANSON, H. (1989). "Etude des Phénomènes d'Entraînement d'Air. Application aux Evacuateurs de Crues." ('A Study of Air Entrainment. Application to Spillway Design') Jl La Houille Blanche, No. 6, pp. 441-462 (en Français).

CHANSON, H. (1992a). "Entraînement d'Air dans les Ecoulements à Surface Libre : Application aux Evacuateurs de Crues de Barrage. " ('Air Entrainment in Open Channel Flow : Application to Spillways.') Jl 'La Houille Blanche', No. 4, 1992, pp. 277-286 (en Français).

CHANSON, H. (1992b). "Discussion of 'A General Correlation for Turbulent Velocity Profiles of Dilute Polymer Solutions', by K.C. TAM, C. TIU, et R.J. KELLER, Jl of Hyd. Res., IAHR, Vol. 30, No. 1, 1992, pp. 117-142." Jl of Hyd. Res., IAHR, Vol. 30, No. 6 (en Anglais).

CHANSON, H. (1992c). "Air Entrainment in Chutes and Spillways." Research Report No. CE 133, Dept. of Civil Engineering, University of Queensland, Australia, Feb., 85 pages (en Anglais).

COMOLET, R. (1979). "Sur le Mouvement d'une bulle de gaz dans un liquide." ('Gas bubble motion in a liquid medium') Jl La Houille Blanche, 1979, No. 1, pp. 31-42 (en Français).

EHRENBERGER, R. (1926). "Wasserbewegung in steilen Rinnen (Susstennen) mit besonderer Berucksichtigung der Selbstbelüftung." ('Flow of Water in Steep Chutes with Special Reference to Selfaeration.') Zeitschrift des Österreichischer Ingenieur und Architektverein, No. 15/16 et 17/18 (en Allemand) (traduit en Anglais par Wilsey, E.F., U.S. Bureau of Reclamation).

EINSTEIN, A. (1906). "Eine Neue Bestimmung der Moleküldimensionen." Ann. Phys., 19, p. 289 (en Allemand).

EINSTEIN, A. (1911). "Eine Neue Bestimmung der Moleküldimensionen." Ann. Phys., 34, p. 591 (en Allemand).

ERVINE, D.A., et FALVEY, H.T. (1987). "Behaviour of Turbulent Water Jets in the Atmosphere and in Plunge Pools." Proc. Instn Civ. Engrs., Part 2, Mar. 1987, 83, pp. 295-314 (en Anglais).

FALVEY, H.T. (1980). "Air-Water Flow in Hydraulic Structures." USBR Engrg. Monograph, No. 41, Denver, Colorado, USA (en Anglais).

HAGER, W.H. (1992). "Spillways, Shockwaves and Air Entrainment - Review and

Recommendations." ICOLD Bulletin, No. 81, Jan. (en Anglais).

HALL, L.S. (1943). "Open Channel Flow at High Velocities." Trans. ASCE, Vol. 108, pp. 1394-1434 (en Anglais).

HARTUNG, F., et SCHEUERLEIN, H. (1970). "Design of Overflow Rockfill Dams." 10th ICOLD Congress, Montréal, Canada, Q. 36, R. 35, pp. 587-598 (en Anglais).

INNEREBNER, K. (1924). "Overflow Channels from Surge Tanks." World Power Conference, 1st, Vol. 2, pp. 481-486 (en Anglais). 
(annexe 2 - suite)

JEVDJEVICH, V., et LEVIN, L. (1953). "Entrainment of Air in flowing Water and Technical Problems connected with it." Proc. of 5th I.A.H.R. Congress, IAHR-ASCE, Minneapolis, USA, pp. 439-454 (en Anglais).

KILLEN, J.M. (1968). "The Surface Characteristics of Self-Aerated Flow in Steep Channels." Ph.D. thesis, University of Minnesota, Minneapolis, USA (en Anglais).

KOSCHITZKY, H.P., et KOBUS, H. (1988). "Hydraulics and Design of Spillway Aerators for Cavitation Prevention in High Speed Flows." Proc. of the Intl Symp. on Hydraulics for High Dams, IAHR, Beijing, China (en Anglais).

LEVIN, L. (1955). "Quelques Réflexions sur la Mécanique de l'écoulement des Mélanges d'Eau et d'Air." ('Notes on the Flow Mechanics of Water-Air Mixtures.') Jl La Houille Blanche, No. 4, Aug.-Sept., 1955, pp. 55-557 (en Français).

LUMLEY, J.L. (1977). "Drag Reduction in Two Phase and Polymer Flows." Physics Fluids, Vol. 20, No. 10, Pt II, pp. S64-S71 (en Anglais).

MADAVAN, N.K., DEUTSCH, S., et MERKLE, C.L. (1984). "Reduction of Turbulent Skin Friction by Microbubbles." Physics Fluids, Vol. 27, No. 2, pp. 356-363 (en Anglais).

MADAVAN, N.K., DEUTSCH, S., et MERKLE, C.L. (1985). "Measurements of Local Skin Friction in a Microbubble-Modified Turbulent Boundary Layer." Jl Fluid Mech., Vol. 156, pp. 237-256 (en Anglais).

MARIE, J.L. (1987). "A Simple Analytical Formulation for Microbubble Drag Reduction." PCH, Vol. 8, No. 2, pp. 213-220 (en Anglais).

MARIE, J.L., MOURSALI, E., et LANCE, M. (1991). "A First Investigation of a Bubbly Boundary Layer on a Flat Plate : Phase Distribution and Wall Shear Stress Measurements." Proc. of the 1st ASMEJSME Fluids Eng. Conf., Turbulence Modification in Multiphase Flows 1991, June, Portland, USA, FEDVol. 110, ASME, pp. 75-80 (en Anglais).

MAY, R.W.P. (1987). "Cavitation in Hydraulic Structures : Occurrence and Prevention." Hydraulics Research Report, No. SR 79, Wallingford, UK (en Anglais).

MICHELS, V., et LOVELY, M. (1953). "Some Prototype Observations of Air Entrained Flow." Proc. of 5th I.A.H.R. Congress, AIHR-ASCE, Minneapolis, USA, pp. 403-414 (en Anglais).

QUINTELA, A.C. (1980). "Flow Aeration to Prevent Cavitation Aeration." Water Power and Dam Construction, 32 (1), Jan., pp. 17-22 (en Anglais).

SENE, K.J. (1984). "Aspects of Bubbly Two-Phase Flow." Ph.D. thesis, Trinity College, Cambridge, UK, Dec. (en Anglais).

STEWART, W.G. (1913). "The Determination of the N in Kutter's Formula for Various Canals, Flumes and Chutes on the Boise Project and Vicinity." Report on 2nd Annual Conf. on Operating Men, USBR, Boise, Idaho, USA, Jan., pp. 8-23 (en Anglais).

STRAUB, L.G., et ANDERSON, A.G. (1958). "Experiments on Self-Aerated Flow in Open Channels." $J l$ of Hyd. Div., Proc. ASCE, Vol. 84, No. HY7, paper 1890 (en Anglais).

TAM, K.C., TIU, C., et KELLER, R.J. (1992). "A General Correlation for Turbulent Velocity Profiles of

Dilute Polymer Solutions." Jl of Hyd. Res., IAHR, Vol. 30, No. 1, pp. 117-142 (en Anglais) VIRK, P.S. (1975). "Drag Reduction Fundamentals." AIChE Jl, Vol. 21, No. 4, pp. 625-656 (en Anglais). VISCHER, D., VOLKART, P., and SIGENTHALER, A. (1982). "Hydraulic Modelling of Air Slots in Open Chutes Spillways." Intl. Conf. on the Hydraulic Modelling of Civil Engineering Structures, BHRA Fluid Engineering, Coventry, UK., pp. 239-252 (en Anglais).

WISNER, P. (1965). "Sur le Rôle du Critère de Froude dans l'Etude de l'Entraînement de l'Air par les Courants a Grande Vitesse." ('On the Role of the Froude Criterion for the Study of Air Entrainment in High Velocity Flows.') Proc. of the 11th IAHR Congress, Leningrad, USSR, paper 1.15. (en Français)

WOOD, I.R. (1983). "Uniform Region of Self-Aerated Flow." Jl Hyd. Eng., ASCE, Vol. 109, No. 3, pp. 447-461 (en Anglais).

WOOD, I.R. (1984). "Air Entrainment in High Speed Flows." Proc. of the Intl. Symp. on Scale Effects in Modelling Hydraulic Structures, IAHR, Esslingen, Germany, H. KOBUS editor, paper 4.1 (en Anglais).

WOOD, I.R. (1991). "Air Entrainment in Free-Surface Flows." LAHR Hydraulic Structures Design Manual No. 4, Hydraulic Design Considerations, Balkema Publ., Rotterdam, Netherlands (en Anglais). 


\section{ANNEXE 3}

\section{Notations}

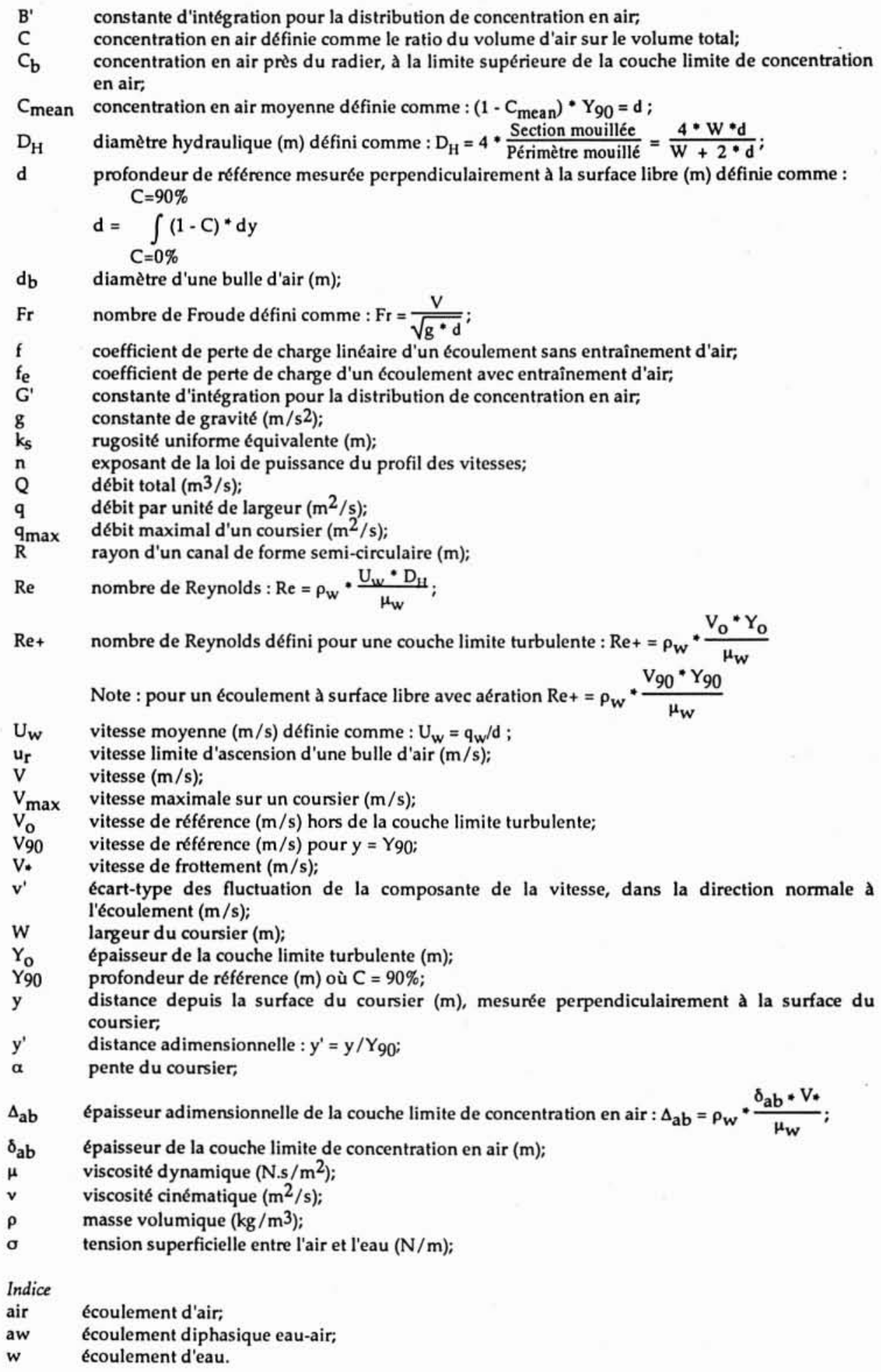


BHR GROUP LIMITED - CONFERENCES, COURSES AND SEMINARS 1993

- 3rd International Conference on

BIOREACTORS \& BIOPROCESSING FLUID DYNAMICS

Cambridge, UK: 14-16 September 1993

Consideration of the biology/fluid dynamics interface of biological processes carried out in industry, and their practical implications for process design and operation.

- Fluid Sealing Technology Short Course

Cranfield, UK: 22-23 September 1993

An intensive introduction to fluid sealing for equipment designers, plant and maintenance engineers, draughtsmen and technical sales engineers.

- 12th International Conference on

SLURRY HANDLING AND PIPELINE TRANSPORT - HYDROTRANSPORT 12

Brugge, Belgium: 28-30 September 1993

A unique meeting-place for representatives of diverse industries to discuss the handling of solid/liquid mixtures, addressing engineering applications and advances in research.

- COST EFFECTIVE PUMP SUMPS

Cranfield, UK: 5 October 1993

A one-day Seminar to include design, the manufacturer's viewpoint, and case studies.

- THE PIPE PROTECTION CONFERENCE

Amsterdam, The Netherlands: 10-12 November 1993

An international forum for operators, designers, contractors and researchers to discuss the most recent developments in methods, materials and legislation, and pinpoint future needs.

- 2nd International Conference on

ADVANCES IN WATER AND EFFLUENT TREATMENT

Cumbria, UK: 8-10 December 1993

Discussion of technologies in treatment management, design, operation and developmnet in the context of tightening standards and legislation.

- EXPERT SYSTEMS 93

Cambridge, UK: 13-15 December 1993

The Annual Technical Conference of the British Computer Society Specialist Group on Expert Systems.

For further details, please contact The Conference Team, BHR Group Limited, Cranfield, Bedford, MK43 OAJ, UK, telephone 0234 750422, fax 0234750074

In addition to organising International Conferences, BHR Group is a leading Technology Centre in Fluid Engineering in Europe and is a world authority in areas such as Mixing, Sealing and Abrasive Water Jetting. A founder of the National Pump Centre, BHR Groups expertise in Pumping and Pipeline Systems is long established with the formation of BHRA in 1947. The Group's Membership Association BHRA continues to supply technical information to members free of charge and to form and maintain strategic links between industry and the leading edge of technology development in BHR Group's wide ranging Fluid Engineering activities. 